\title{
An Adaptive Binarization Technique for Low Quality Historical Documents
}

\author{
Basilios Gatos, Ioannis Pratikakis, and Stavros J. Perantonis \\ Computational Intelligence Laboratory, \\ Institute of Informatics and Telecommunications, \\ National Research Center "Demokritos", \\ 15310 Athens, Greece \\ \{bgat, ipratika, sper\}@iit.demokritos.gr \\ http://www.iit.demokritos.gr/cil
}

\begin{abstract}
Historical document collections are a valuable resource for human history. This paper proposes a novel digital image binarization scheme for low quality historical documents allowing further content exploitation in an efficient way. The proposed scheme consists of five distinct steps: a pre-processing procedure using a low-pass Wiener filter, a rough estimation of foreground regions using Niblack's approach, a background surface calculation by interpolating neighboring background intensities, a thresholding by combining the calculated background surface with the original image and finally a post-processing step in order to improve the quality of text regions and preserve stroke connectivity. The proposed methodology works with great success even in cases of historical manuscripts with poor quality, shadows, nonuniform illumination, low contrast, large signal-dependent noise, smear and strain. After testing the proposed method on numerous low quality historical manuscripts, it has turned out that our methodology performs better compared to current state-of-the-art adaptive thresholding techniques.
\end{abstract}

\section{Introduction}

It is common that documents belonging to historical collections are poorly preserved and prone to degradation processes. This work aims at leveraging state-of-the-art techniques in digital image binarization and text identification for digitized documents allowing further content exploitation in an efficient way. The method has been developed in the framework of the Greek GSRT-funded R\&D project, D-SCRIBE, which aims to develop an integrated system for digitization and processing of old Greek manuscripts.

Binarization (threshold selection) is the starting step of most document image analysis systems and refers to the conversion of the gray-scale image to a binary image. Binarization is a key step in document image processing modules since a good binarization sets the base for successful segmentation and recognition of characters. In old document processing, binarization usually distinguishes text areas from background areas, so it is used as a text locating technique. In the literature, the binariza- 
tion is usually reported to be performed either globally or locally. The global methods (global thresholding) use a single threshold value to classify image pixels into object or background classes [1-5], whereas the local schemes (adaptive thresholding) can use multiple values selected according to the local area information [6,7]. Most of the proposed algorithms for optimum image binarization rely on statistical methods, without taking into account the special nature of document images [8-10]. Global thresholding methods are not sufficient for document image binarization since document images usually have poor quality, shadows, nonuniform illumination, low contrast, large signal-dependent noise, smear and strains. Instead, adaptive to local information techniques for document binarization have been developed [11-14].

In this paper, a novel adaptive thresholding scheme is introduced in order to binarize low quality historical documents and locate meaningful textual information. The proposed scheme consists of five basic steps. The first step is dedicated to a denoising procedure using a low-pass Wiener filter. We use an adaptive Wiener method based on statistics estimated from a local neighborhood of each pixel. In the second step, we use Niblack's approach for a first rough estimation of foreground regions. Usually, the foreground pixels are a subset of Niblack's result since Niblack's method usually introduces extra noise. In the third step, we compute the background surface of the image by interpolating neighboring background intensities into the foreground areas that result from Niblack's method. A similar approach has been proposed for binarizing camera images [15]. In the fourth step, we proceed to final thresholding by combining the calculated background surface with the original image. Text areas are located if the distance of the original image from the calculated background exceeds a threshold. This threshold adapts to the gray-scale value of the background surface in order to preserve textual information even in very dark background areas. In the final step, a post-processing technique is used in order to eliminate noise pixels, improve the quality of text regions and preserve stroke connectivity. The proposed method was tested with a variety of low quality historical manuscripts and it turned out that it is superior to current state-of-the-art adaptive thresholding techniques.

\section{Previous Work}

Among the most known approaches for adaptive thresholding is Niblack's method [8] and Sauvola's method [11].

Niblack's algorithm [8] calculates a pixelwise threshold by shifting a rectangular window across the image. The threshold $T$ for the center pixel of the window is computed using the mean $m$ and the variance $s$ of the gray values in the window:

$$
T=m+k s
$$

where $k$ is a constant set to -0.2 . The value of $k$ is used to determine how much of the total print object boundary is taken as a part of the given object. This method can distinguish the object from the background effectively in the areas close to the objects. The results are not very sensitive to the window size as long as the window covers at least 1-2 characters. However, noise that is present in the background remains domi- 
nant in the final binary image. Consequently, if the objects are sparse in an image, a lot of background noise will be left. Sauvola's method [11] solves this problem by adding a hypothesis on the gray values of text and background pixels (text pixels have gray values near 0 and background pixels have gray values near 255), which results in the following formula for the threshold:

$$
T=m+(1-k(1-s / R))
$$

where $R$ is the dynamic range of the standard deviation fixed to 128 and $k$ is fixed to 0.5. This method gives better results for document images.

\section{Methodology}

The proposed methodology for low quality historical document binarization and text preservation is illustrated in Fig. 1 and fully described in this section.

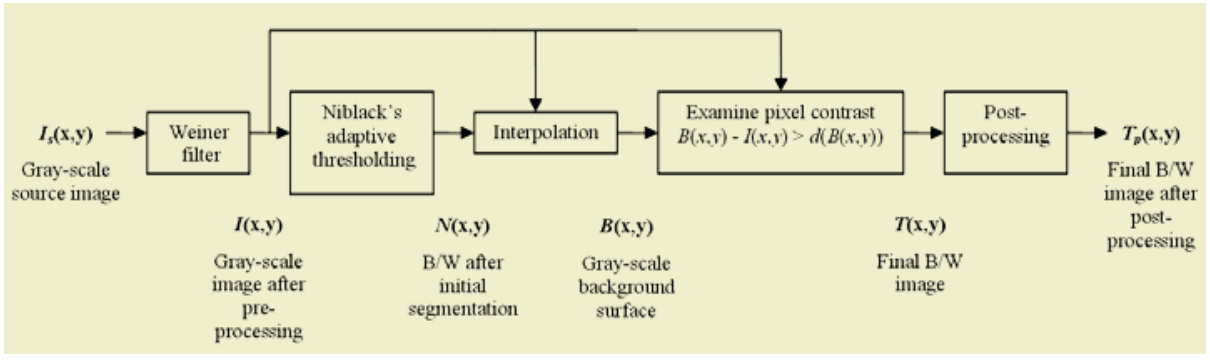

Fig. 1. Block diagram of the proposed methodology for low quality historical document text preservation.

\subsection{Stage1: Pre-processing}

Since historical document collections are usually of very low quality, a pre-processing stage of the grayscale source image is essential in order to eliminate noise areas, to smooth the background texture and to highlight the contrast between background and text areas. The use of a low-pass Wiener filter [16] has proved efficient for the above goals. Wiener filter is commonly used in filtering theory for image restoration. Our pre-processing module implements an adaptive Wiener method based on statistics estimated from a local neighborhood around each pixel. The grayscale source image $I_{s}$ is transformed to grayscale image $I$ according to the following formula:

$$
I(x, y)=\mu+\left(\sigma^{2}-v^{2}\right)\left(I_{s}(x, y)-\mu\right) / \sigma^{2}
$$

where $\mu$ is the local mean and $\sigma^{2}$ the variance at a $N x M$ neighborhood around each pixel. We used a 3x3 Wiener filter for documents with 1-2 pixel wide characters otherwise we used a $5 \times 5$ kernel. Fig. 2 shows the results of applying a 3x3 Wiener filter to a document image. 


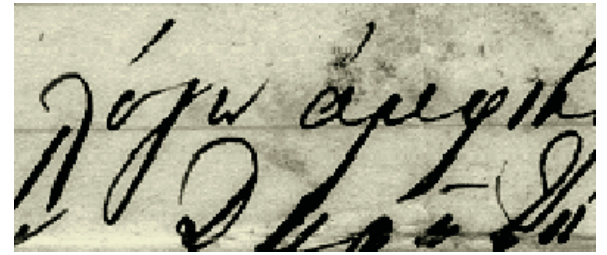

(a)

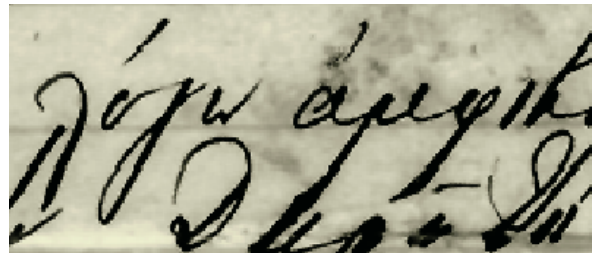

(b)

Fig. 2. Image pre-processing: (a) Original image; (b) 3x3 Wiener filter.

\subsection{Stage2: Rough Estimation of Foreground Regions}

At this step of our approach, we want to obtain a rough estimation of foreground regions. Our intention is to proceed to an initial segmentation to foreground and background regions and find a set of foreground pixels that is a superset of the correct set of foreground pixels. In other words, we intend to obtain a set of pixels that contains the foreground pixels plus some noise. Niblack's approach for adaptive thresholding [8] is suitable for this case since Niblack's method usually detects text regions but introduces extra noise (see Fig. 3). At this step, we process image $I(x, y)$ in order to extract the binary image $N(x, y)$ that has 1 's for the rough estimated foreground regions.

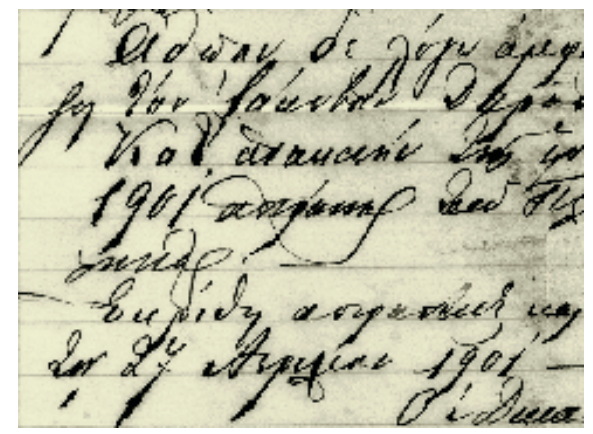

(a)

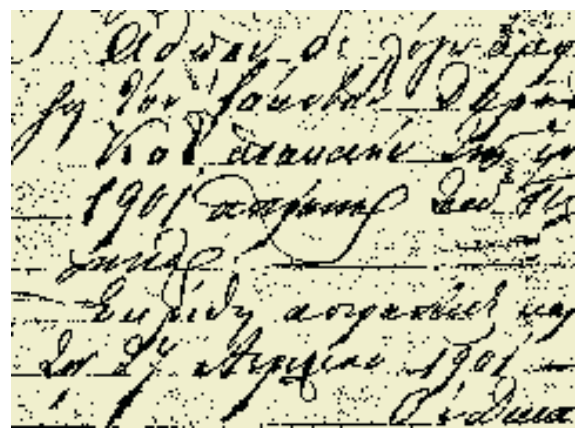

(b)

Fig. 3. Adaptive thresholding using Niblack's approach: (a) Original image; (b) Estimation of foreground regions.

\subsection{Stage3: Background Surface Estimation}

At this stage, we compute an approximate background surface $B(x, y)$ of the image. The pixels of the pre-processed source image $I(x, y)$ belong to the background surface $B(x, y)$ only if the corresponding pixels of the resulting rough estimated foreground image $N(x, y)$ have zero values. The remaining values of surface $B(x, y)$ are interpolated from neighboring pixels. The formula for $B(x, y)$ calculation is as follows: 


$$
B(x, y)=\left\{\begin{array}{cc}
I(x, y) & \text { if } N(x, y)=0 \\
\frac{\sum_{i=x-d x}^{x+d x} \sum_{j=y-d y}^{y+d y}(I(i, j)(1-N(i, j)))}{\sum_{i=x-d x}^{x+d x} \sum_{j=y-d y}^{y+d y}(1-N(i, j))} & \text { if } N(x, y)=1
\end{array}\right.
$$

The interpolation window of size $d x \mathrm{x} d y$ is defined to cover at least two image characters. An example of the background surface estimation is demonstrated in Fig. 4.

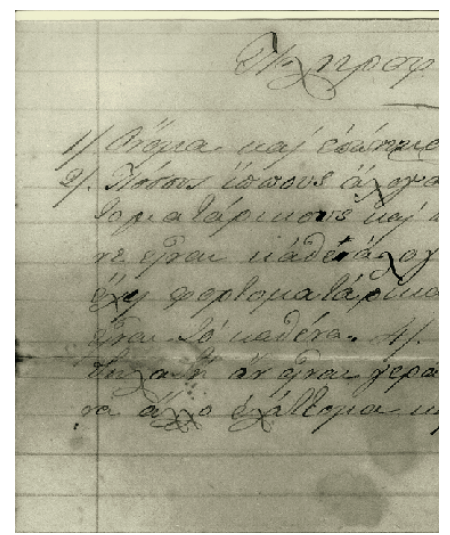

(a)

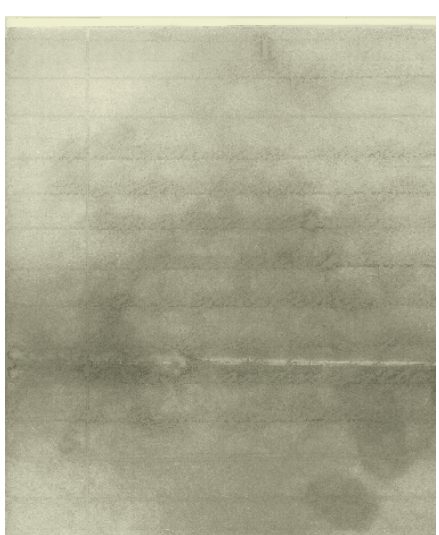

(b)

Fig. 4. Background surface estimation: (a) Original image $I$; (b) Background surface $B$.

\subsection{Stage4: Final Thresholding}

In this step, we proceed to final thresholding by combining the calculated background surface $B$ with the original image $I$. Text areas are located if the distance of the original image from the calculated background is over a threshold $d$. We suggest that the threshold $d$ must change according to the gray-scale value of the background surface $B$ in order to preserve textual information even in very dark background areas. For this reason, we propose a threshold $d$ that has smaller values for darker regions. The final binary image $T$ is given by the following formula:

$$
T(x, y)= \begin{cases}1, & \text { if } B(x, y)-I(x, y)>d(B(x, y)) \\ 0, & \text { otherwise }\end{cases}
$$

A typical histogram of a document image (see Fig. 5) has two peaks, one for text regions and one for background regions. The average distance $\delta$ between the foreground and background can be calculated by the following formula: 


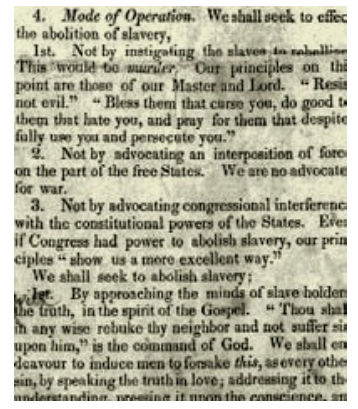

(a)

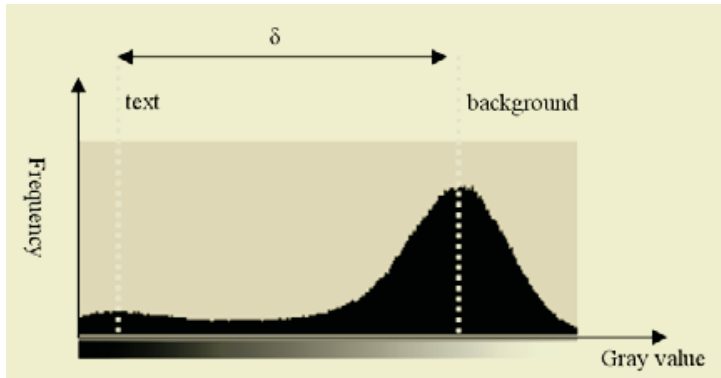

(b)

Fig. 5. Document image histogram: (a) Original image; (b) Gray level histogram.

$$
\delta=\frac{\sum_{x} \sum_{y}(B(x, y)-I(x, y))}{\sum_{x} \sum_{y} N(x, y)}
$$

For the usual case of document images with uniform illumination, the minimum threshold $d$ between text pixels and background pixels can be defined with success as $q \cdot \delta$ where $q$ is a variable near 0.8 that helps preserving the total character body in order to have successful OCR results [15]. In the case of very old documents with low quality and nonuniform illumination we want to have a smaller value for the threshold $d$ for the case of darker regions since it is a usual case to have text in dark regions with small foreground-background distance. To achieve this, we first compute the average background values $b$ of the background surface $B$ that correspond to the text areas of image $N$ :

$$
b=\frac{\sum_{x} \sum_{y}(B(x, y)(1-N(x, y)))}{\sum_{x} \sum_{y}(1-N(x, y))}
$$

We wish the threshold to be approximately equal to the value $q \cdot \delta$ when the background is large (roughly greater than the average background value $b$ ) and approximately equal to $p_{2} \cdot q \cdot \delta$ when the background is small (roughly less than $p_{1} \cdot b$ ) with $p_{1}, p_{2} \in[0,1]$. To simulate this desired behaviour, we use the following logistic sigmoid function that exhibits the desired saturation behaviour for large and small values of the background as shown in Fig. 6:

$$
d(B(x, y))=q \delta\left(\frac{\left(1-p_{2}\right)}{1+\exp \left(\frac{-4 B(x, y)}{b\left(1-p_{1}\right)}+\frac{2\left(1+p_{1}\right)}{\left(1-p_{1}\right)}\right)}+p_{2}\right)
$$




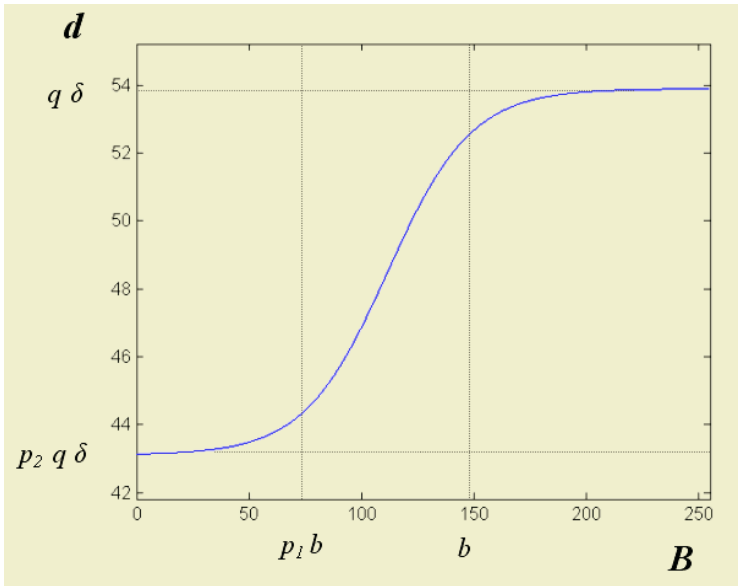

Fig. 6. Function $d(B(x, y))$.

After experimental work, for the case of old manuscripts, we suggest the following parameter values: $q=0.6, p_{1}=0.5, p_{2}=0.8$.

\subsection{Stage5: Post-processing}

In the final step, we proceed to post-processing of the resulting $\mathrm{b} / \mathrm{w}$ image in order to eliminate noise, improve the quality of text regions and preserve stroke connectivity by isolated pixel removal and filling of possible breaks, gaps or holes. Below follows a detailed step-by-step description of the post-processing algorithm that consists of a consequent application of shrink and swell filtering [17].

Step 1: A shrink filter is used to remove noise from the background. The entire $\mathrm{b} / \mathrm{w}$ image is scanned and each foreground pixel is examined. If $P_{s h}$ is the number of background pixels in a sliding $n \times n$ window, which has the foreground pixel as the central pixel, then this pixel is changed to background if $P_{s h}>k_{s h}$ where $k_{s h}$ can be defined experimentally.

Step 2: A swell filter is used to fill possible breaks, gaps or holes in the foreground. The entire $\mathrm{b} / \mathrm{w}$ image is scanned and each background pixel is examined. If $P_{s w}$ is the number of foreground pixels in a sliding $n \times n$ window, which has the background pixel $(x, y)$ as the central pixel, and $x_{a}, y_{a}$ the average values for all foreground pixels in the $n \times n$ window, then this pixel is changed to foreground if $P_{s w}>k_{s w}$ and $\left|x-x_{a}\right|<d x$ and $\left|\mathrm{y}-y_{a}\right|<d y$. The latter two conditions are used in order to prevent an increase in the thickness of character strokes since we examine only background pixels among uniformly distributed foreground pixels.

Step 3: An extension of the above conditions, leads to a further application of a swell filter that is used to improve the quality of the character strokes. The entire $\mathrm{b} / \mathrm{w}$ image is scanned and each background pixel is examined. If $P_{s w l}$ is the number of foreground 
pixels in a sliding $n \mathrm{x} n$ window, which has the background pixel as the central pixel, then this pixel is changed to foreground if $P_{s w l}>k_{s w l}$.

After experimental work, for the case of old manuscripts with an average letter height of $l_{h}$, we suggest the following parameter values for the post-processing phase: $n=0.15 l_{h}, \quad k_{s h}=0.9 n^{2}, k_{s w}=0.05 n^{2}, d x=d y=0.25 n, k_{s w l}=0.35 n^{2}$. An example of a resulting $\mathrm{b} / \mathrm{w}$ image after post-processing steps is given in Fig. 7.

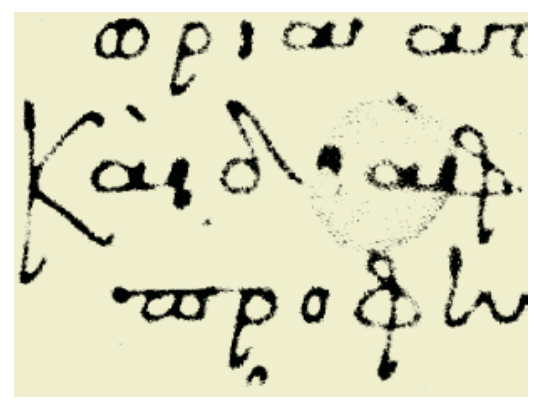

(a)

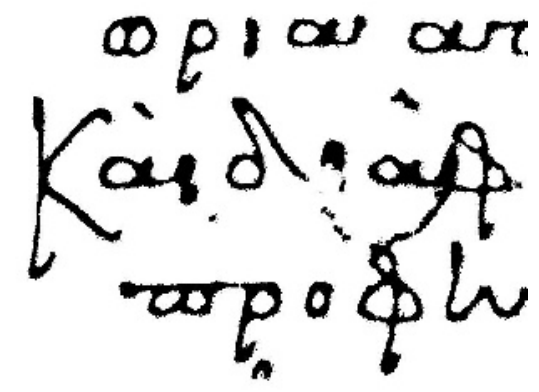

(c)

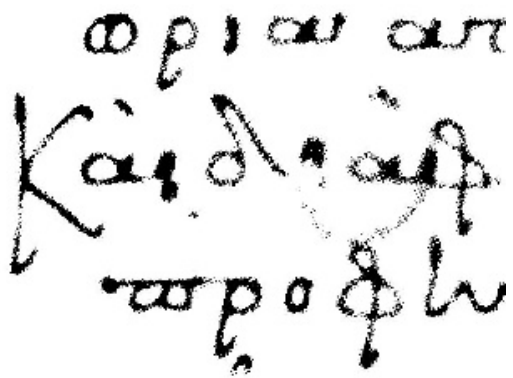

(b)

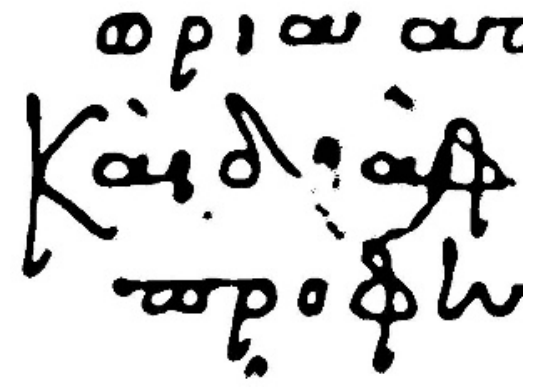

(d)

Fig. 7. Post-processing stage: (a) B/W image resulting after final thresholding stage; (b) Image after post-processing step 1; (c) Image after post-processing step 2; (d) Final image after postprocessing.

\section{Experimental Results}

In this section, we compare the performance of our algorithm with those of Otsu [2], Niblack [8] and Sauvola et al. [11]. The testing set includes images from both handwritten and typed historical manuscripts. All images are of poor quality and have shadows, nonuniform illumination, smear and strain. Fig. 8 demonstrates an example of a typed manuscript, while Fig. 9, 10 demonstrate examples of handwritten manuscripts. The final post-processing task is demonstrated at Fig. 10. As shown in all cases, our algorithm out-performs all the rest of the algorithms in preservation of meaningful textual information. 


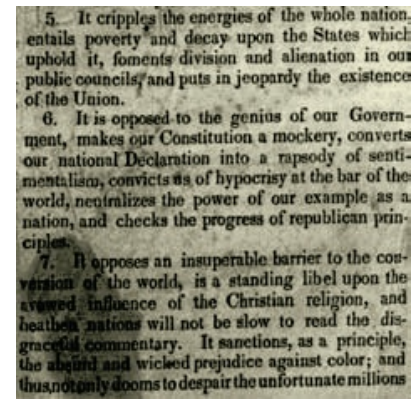

(a)
15. It cripples the energies of the whole nation entails poverty and decay upon the States which uphold it, foments division and alienation in ous public councilsf and puts in jeopardy the existence of the Union. 6. It is opposed to the genius of our Govern-
ment, 'makes ogr Constitution a mockery, converts our nationar becclaration into a rapsody of sentimentalisis, convicts ts of hypocrisy at the bar of the world; neuinalizes the power of our example, as a nation, and checks the progress of republican prinIf It cripples the energies of the whole nation. It cripples the encrgies of the whole nation.
entails poverty and decay upon the States whict, entails poverty' and decay upon the States whict uphold it, foments division and alienation in ou upbold it, foments division and alienation in ous public councilsfand puts in jeopardy the existence, public councils and puts in jeopardy the existence public councils and puts in jeopardy the existence; public councily and puts in jeopardy the existence Union.
of the Union. (6. It is opposed to the genius of our Govern- 6. It is opposed to the genius of our Government,' 'makes ojir Constitution a mockery, converts ment, 'makes our 'Constitution a mockery, converts our' nationar Déclaration into a rapeody of senti- our, nationat Declaration into a rapsody of sentimentaliśn, 'convivis \&s of hypocrisy at the bar of the mentalism, convicts ts of hypocrisy at the bar of the world; neufralizes the power of our example as a world, nentralizes the power of our example, as a niation, and checks the progress of republican prin- nation, and checks the progress of republican prin. nation, and checks the progress of republican prin- nat

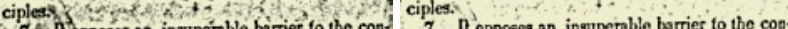
7. A Opposes an insuperable barrier to the con- 7. It opposes an insuperable barrier to the conyersion of the world is a standing libel upon the version of the world, is a standing libel upon the asewed influence of the Christian religion, and avowed influence of the Christian religion, and

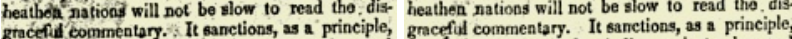
the abaifrd and wicleed prejudice against color; and the abgurd and wicled prejudice against color; and thusnot tonly dooms to despair the unfortunate millions, thus,notonly dooms to despair the unfortunatemillions

$$
\text { (c) }
$$

5. It cripples the energies of the whole nation, entails poverty and decay upon the States which uphold it, foments division and alienation in ou existence the Union.

6. It is opposed to the genius of our Government, ' makes our Constitution a mockery, converts our, nationar Declaration into a rapeody of sentimentalism, convicts ts of hypocrisy at the bar of the world, nentralizes the power of our example as a nation, and checks the progress of republican prin. ciples:

7. It opposes an insuperable barrier to the convergion of the world, is a standing libel upon the arowed influence of the Christian religion, and heathea nations will not be slow to read the disgracefiul commentary. It sametions, as a principle, the abyurd and wiched prejudice against color; and thus,notonily dooms to despair the unfortunate millions

Fig. 8. Experimental results - binarization of a typed manuscript (a) Original image; (b) Otsu's method; (c) Niblack's method; (d) Sauvola's method; (e) The proposed method (without postprocessing).

In order to extract some quantitative results for the efficiency of the proposed binarization method, we compared the results obtained by the well-known OCR engine FineReader 6 [18] with and without applying the proposed binarization technique, as well as with applying the binarization techniques of Niblack [8] and of Sauvola et al. [11]. To measure the quality of the OCR results we calculated the Levenshtein distance [19] between the correct text (ground truth) and the resulting text. As shown at table 1 , in all cases, the recognition results were improved after applying the proposed binarization technique. The application of the other two binarization techniques results in worse results in most cases.

Table 1. Representative OCR results after applying several binarization schemes.

\begin{tabular}{|l|c|c|c|c|}
\hline & \multicolumn{4}{|c|}{ Levenshtein Distance from the Ground truth } \\
\hline & Document 1 & Document 2 & Document 3 & Document 4 \\
\hline Original image & 68 & 221 & 185 & 408 \\
\hline Niblack's method & 228 & 619 & 513 & 447 \\
\hline Sauvola's method & 60 & 394 & 276 & 694 \\
\hline The proposed method & 56 & 207 & 177 & 153 \\
\hline
\end{tabular}




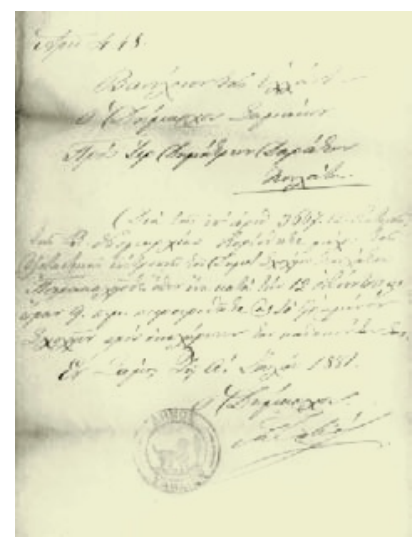

(a)

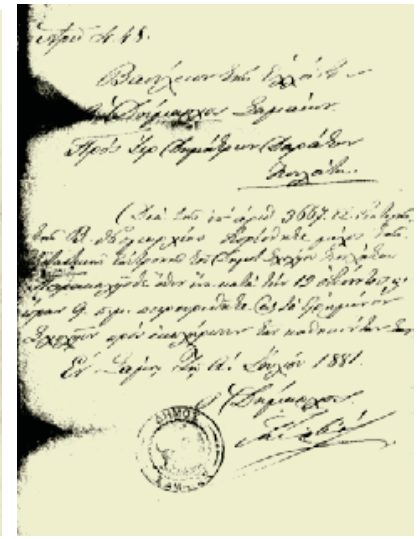

(b)

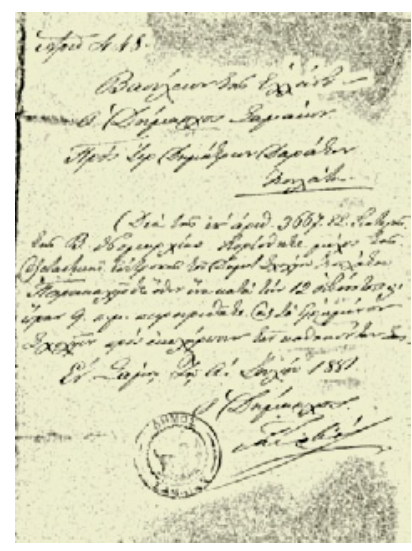

(c)

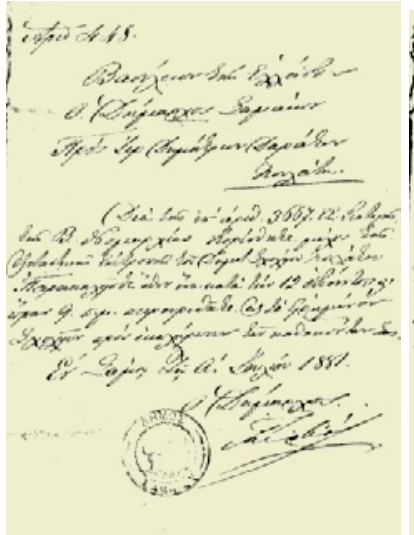

(d)

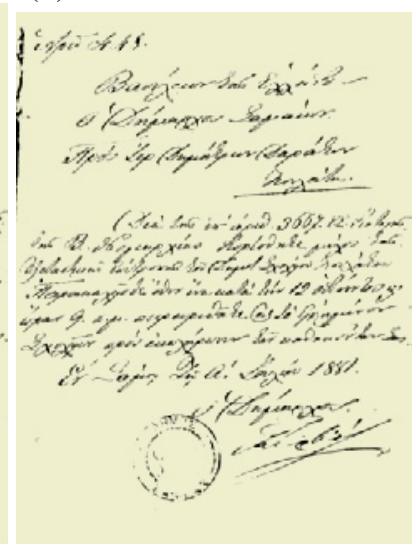

(e)

Fig. 9. Experimental results - binarization of a handwritten manuscript (a) Original image; (b) Otsu's method; (c) Niblack's method; (d) Sauvola's method; (e) The proposed method (without post-processing).

\section{Conclusions}

In this paper, we present a novel methodology that leads to preservation of meaningful textual information in low quality historical documents. The proposed scheme consists of five distinct steps: a pre-processing procedure using a low-pass Wiener filter, a rough estimation of foreground regions using Niblack's approach, a background surface calculation by interpolating neighboring background intensities, a thresholding by combining the calculated background surface with the original image and finally a post-processing technique in order to eliminate noise pixels, improve the quality of text regions and preserve stroke connectivity. Text areas are located if the distance of the original image with the calculated background is over a threshold. This threshold adapts to the gray-scale value of the background surface in order to preserve textual 
information even in very dark background areas. The proposed methodology works with great success even in cases of historical manuscripts with poor quality, shadows, nonuniform illumination, low contrast, large signal-dependent noise, smear and strain. Experimental results show that our algorithm outperforms the most known thresholding approaches.

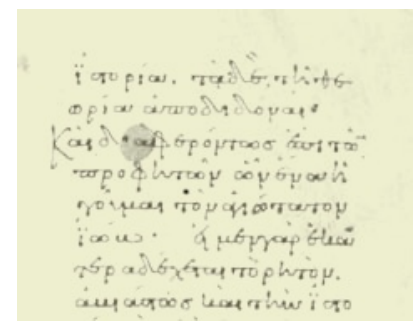

(a)

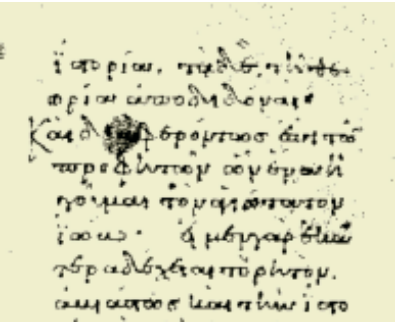

(b)

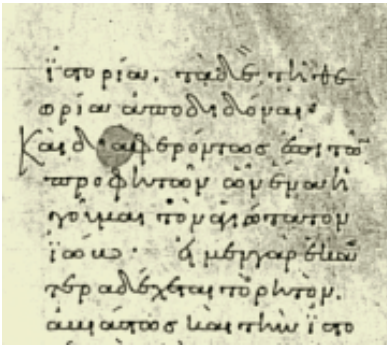

(c)

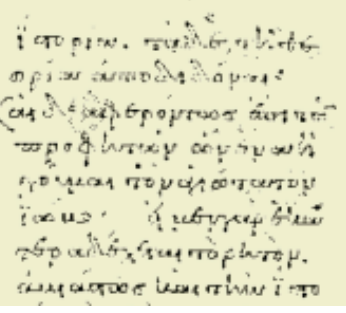

(d)

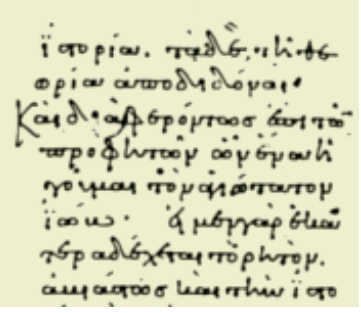

(e)

Fig. 10. Experimental results - binarization of a handwritten manuscript: (a) Original image; (b) Otsu's method; (c) Niblack's method; (d) Sauvola's method; (e) The proposed method (including post-processing).

\section{Acknowledgment}

The authors would like to thank Dr. Ergina Kavallieratou for providing us samples from her private historical document collection.

This work is supported by the Greek GSRT-funded R\&D project, D-SCRIBE, which aims to develop an integrated system for digitization and processing of old Greek manuscripts.

\section{References}

1. Rosenfeld, A., Kak, A. C.: Digital Picture Processing, $2^{\text {nd }}$ edition, Academic Press, New York (1982)

2. Otsu, N.: A threshold selection method from gray-level histograms. IEEE Trans. Systems Man Cybernet. 9 (1) (1979) 62-66 
3. Kittler, J., Illingworth, J.: On threshold selection using clustering criteria. IEEE Trans. Systems Man Cybernet. 15 (1985) 652-55

4. Brink, A. D.: Thresholding of digital images using two-dimensional entropies. Pattern Recognition 25 (8) (1992) 803-808

5. Yan, H.: Unified formulation of a class of image thresholding techniques. Pattern Recognition 29(12) (1996) 2025-2032

6. Sahoo, P. K., Soltani, S., Wong, A. K. C.: A survey of Thresholding Techniques. Computer Vision, Graphics and Image Processing, 41(2) (1988) 233-260

7. Kim, I. K., Park, R. H.: Local adaptive thresholding based on a water flow model. Second Japan-Korea Joint Workshop on Computer Vision, Japan (1996) 21-27

8. Niblack, W.: An Introduction to Digital Image Processing. Englewood Cliffs, N. J., Prentice Hall (1986) 115-116

9. Yang, J., Chen, Y., Hsu, W.: Adaptive thresholding algorithm and its hardware implementation. Pattern Recognition Lett. 15(2) (1994) 141-150

10. Parker, J. R., Jennings, C., Salkauskas, A. G.: Thresholding using an illumination model. ICDAR'93 (1993) 270-273

11. Sauvola, J., Pietikainen, M.: Adaptive Document Image Binarization. Pattern Recognition 33(2000), 225-236

12. Chang, M., Kang, S.,Rho, W., Kim, H., Kim, D.: Improved binarization algorithm for document image by histogram and edge detection. ICDAR'95 (1993) 636-643

13. Trier, O. D., Jain, A. K.: Goal-Directed Evaluation of Binarization Methods. IEEE Trans. on Patt. Anal. and Mach. Intell., 17(12) (1995) 1191-1201

14. Eikvil, L., Taxt, T., Moen, K.: A fast adaptive method for binarization of document images. Int. Conf. Document Analysis and Recognition, France (1991) 435-443

15. Seeger, M., Dance, C.: Binarising Camera Images for OCR. Sixth International Conference on Document Analysis and Recognition (ICDAR'01), Seattle, Washington (2001) 54-58

16. Jain, A.: Fundamentals of Digital Image Processing. Prantice Hall, Englewood Cliffs, NJ (1989)

17. Schilling, R. J.: Fundamentals of Robotics Analysis and Control. Prentice-Hall, Englewood Cliffs, NJ (1990)

18. www.finereader.com

19. Levenshtein, V. I.: Binary codes capable of correcting deletions, insertions and reversals. Sov. Phys. Dokl., 6 (1966) 707-710 